\title{
Structural, Magnetic, and Electronic Properties of Fe: A Screened Hybrid Functional Study
}

\author{
Young-Rok Jang ${ }^{1}$ and Byung Deok Yu ${ }^{2 *}$ \\ ${ }^{1}$ Department of Physics, University of Incheon, Incheon 406-772, Korea \\ ${ }^{2}$ Department of Physics, University of Seoul, Seoul 130-743, Korea
}

(Received 20 July 2011, Received in final form 30 August 2011, Accepted 8 September 2011)

\begin{abstract}
We performed total energy and electronic structure calculations for the basic ground state properties of Fe using the conventional generalized gradient approximation (GGA) and screened hybrid functionals as the form of the exchange-correlation functional. To that end, we calculated structural (equilibrium lattice constants, bulk moduli, and cohesive energies) and electronic (magnetic moments and densities of states) properties. Both functional calculations gave the correct ground state, the ferromagnetic bec phase, in which the structural parameters agreed well with experimental results. However, the description of the cohesive energies and magnetic moments at the ground state exhibited different behavior from each other: the unusually small cohesive energy and large magnetic moment were observed in the screened hybrid functional calculations compared to the GGA calculations. The reason for the difference was examined by analyzing the calculated electronic structures.
\end{abstract}

Keywords : density functional theory, hybrid functional, Fe, ground state properties, electronic properties

\section{Introduction}

Electronic structure calculation methods based on density functional theory (DFT) have been used extensively to determine material properties in condensed matter physics as well as in chemistry $[1,2]$. For DFT calculations, proper selection of exchange-correlation (XC) functional should be made to obtain reliable results that are compatible with experimental ones. The most common approximation was implemented within the local-density approximation (LDA), which has been very successful in many applications. However, the LDA fails for some systems, such as transition metals. Following the "Jacob's ladder" of the XC functional, introduced by Perdew [3], the second rung above the LDA was implemented by introducing gradient corrections, which led to the generalized gradient approximation (GGA) [4, 5]. This gradient correction approach has resulted in considerably better agreement between experiment and theory for the basic properties of transition metals [6-14]. For instance, the GGA provides the correct results for the ferromagnetic

*Corresponding author: Tel: +82-2-2210-2639

Fax: +82-2-2245-6531, e-mail: ybd@uos.ac.kr
(FM) bcc ground state for $\mathrm{Fe}$, while the ground state of the LDA is the nonmagnetic (NM) hep phase [10-12]. However, the LDA or GGA based on the DFT fails in the description of band gaps of semiconductors and insulators [15]. A significant step forward for correcting band gaps was achieved with the introduction of so-called hybrid functionals, such as HSE06 [16, 17] and PBE0 [18], obtained by a combination of exact nonlocal Hartree-Fock exchange and a standard DFT exchange. These functionals have represented a significant improvement over the LDA or GGA for molecules as well as extended semiconductors and insulators [15].

Despite these efforts, a comprehensive hybrid functional study for a set of diverse solids is still lacking. For instance, in recent years, metal-supported ultrathin oxide films have attracted considerable attention due to their importance in many technological applications, such as catalysis, microelectronics, and magnetic devices [19-25]. To apply a hybrid functional to oxide/metal systems, we need to check the validity of the hybrid functional for transition metals, particularly, itinerant magnetic systems. Recently, it was reported that, in the description of cohesive energies and magnetic properties of $d$ metals, the hybrid functional underperforms compared with the GGA 
functional [26]. Nevertheless, a detailed understanding of the structural properties and associated electronic structures for itinerant magnetic systems remains uncertain. In this study, we performed electronic structure calculations for a detailed evaluation of the description of the ground state properties of Fe using the GGA and hybrid functionals. A comparative study was done by investigating the structural and electronic properties of $\mathrm{Fe}$.

This paper is organized as follows. In Sec. 2, the computational method and settings that were used in this study are described briefly. The results and discussion are presented in Sec. 3. Finally, our findings are summarized in Sec. 4.

\section{Computation Method}

All spin-polarized calculations were performed using the DFT as implemented in the Vienna Ab initio Simulation Package (VASP) code $[27,28]$. The generalizedgradient correction for the XC functional was approximated with the Perdew-Burke-Ernzerhof (PBE) expression [5]. Screened hybrid functional calculations were performed using the HSE06 functional [16, 17], in which the XC energy $E_{\mathrm{XC}}$ is expressed as

$$
E_{\mathrm{XC}}^{\mathrm{HSE}}=\alpha E_{\mathrm{X}}^{\mathrm{sr}}(\mu)+(1-\alpha) E_{\mathrm{X}}^{\mathrm{PBE}, \mathrm{lr}}(\mu)+E_{\mathrm{C}}^{\mathrm{PBE}}
$$

Here the exchange component of the electron-electron interaction is separated into a short-range (sr) and a longrange (lr) part using the construction

$$
\frac{1}{r}=\operatorname{sr}_{\mu}(r)+\operatorname{lr}_{\mu}(r)=\frac{1-\operatorname{erf}(\mu r)}{r}+\frac{\operatorname{erf}(\mu r)}{r} .
$$

The exchange part of the XC energy, $E_{\mathrm{XC}}$, is constructed by mixing a fraction $\alpha$ of the short-range nonlocal Fock exchange energy $E_{\mathrm{X}}^{\mathrm{sr}}(\mu)$ with the short-range PBE exchange energy $E_{\mathrm{X}}^{\mathrm{PBE}, \mathrm{sr}}$. In the context of the HSE06 functional, the optimum screening parameter $\mu$ is approximately $0.2 \AA^{-1}$ and the mixing fraction $\alpha$ is 0.25 .

For electron-ion interactions, the projector augmentedwave (PAW) method [29] was used. The electronic wave functions were expanded by plane waves with a kineticenergy cutoff of $380 \mathrm{eV}$. In the PBE calculations, a $\Gamma$ centered $16 \times 16 \times 16 \mathrm{k}$-point mesh in the Brillouin zone (BZ) of the primitive cell was used for the bcc and fcc phases, and a $16 \times 16 \times 8$ k-point mesh was used for the hcp phase. In the HSE06 calculations, the computational cost of evaluating the Fock exchange scales quadratically with the number of $\mathbf{k}$ points. To reduce the computational load in the HSE06 calculations, we used a $10 \times 10 \times 10$ mesh for the bcc and fcc phases, and a $10 \times 10 \times 5$ mesh for the hcp phase. The k-point convergence was checked by performing calculations using a larger $12 \times 12 \times 12$ mesh for the ferromagnetic bcc phase. This convergence test in the HSE06 calculations suggests that the calculated lattice constant, bulk modulus, cohesive energy, and magnetic moment are accurate up to $0.003 \AA, 5 \mathrm{GPa}, 0.01 \mathrm{eV}$, and $0.01 \mu_{\mathrm{B}}$, respectively. Thus, the computational accuracy is sufficient for the purposes of our study.

\section{Results and Discussion}

The static structural properties are determined by calculating the total energy as a function of the volume and fitting to some equation-of-state function. In the present study, we used the Birch-Murnaghan (B-M) form [30], which is one of the most commonly used fitting functions. The theoretical ground-state lattice constant for each functional was given as the minimum point of the fitted total energy.

First we performed total energy calculations for the ground-state properties of $\mathrm{Fe}$ using the PBE GGA functional. The energy-volume curves for various structures of $\mathrm{Fe}$ are shown in Fig. 1. We found that the PBE GGA calculations gave the correct ground state, the FM bcc structure, in good agreement with previous studies $[6,10$, 11,31]. For the FM bcc phase, we also investigated the

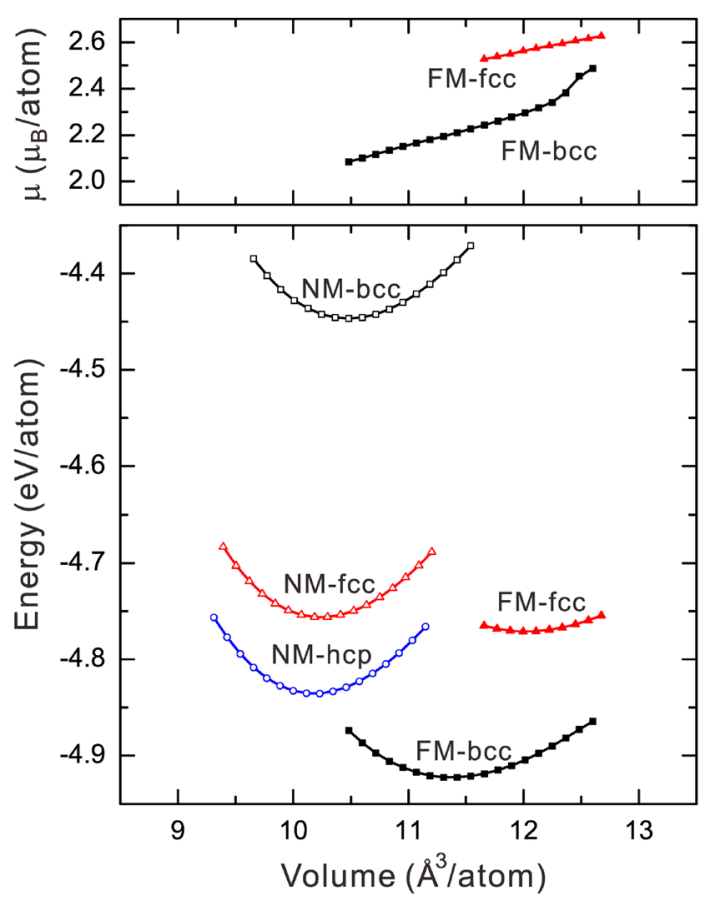

Fig. 1. (Color online) Magnetic moments and total energies as a function of volume for bcc, fcc, and hcp Fe in the nonmagnetic (NM) and ferromagnetic (FM) phases from PBE calculations. It is noted that the FM phase of hcp Fe was unstable in the PBE calculations. 
magnetic moment as a function of the volume (see Fig. 1). A pronounced jump in the magnetic moment was obtained as observed in previous works [11, 32]. Zhang et al. suggested that such a jump influenced the B-M fits on the data points [32]. For the present B-M fits, we used the data points in the volume region with such a jump in the magnetic moment. The calculated equilibrium lattice constant, bulk modulus, magnetic moment, and cohesive energy are summarized in Table 1 . The calculated results $\left(a_{0}=2.833 \AA, B_{0}=177 \mathrm{GPa}\right.$, and $\left.\mu=2.20 \mu_{\mathrm{B}}\right)$ were very close to those of the very recent theoretical results from the all-electron full-potential linearized augmented planewave (FLAPW) method based on the DFT $\left(a_{0}=2.831 \AA\right.$, $B_{0}=173 \mathrm{GPa}$, and $\mu=2.18 \mu_{\mathrm{B}}$ ) [11]. The magnetization energy $E_{\mathrm{mag}}$ in the bcc phase (defined as the difference between the total energies of the FM and the NM states) was $0.48 \mathrm{eV}$, which was very close to the previous results $\left(E_{\mathrm{mag}}=0.48 \mathrm{eV}\right.$ [31] and $\left.0.52 \mathrm{eV}[6]\right)$.

Next, the screened hybrid HSE06 functional calculations were performed. Fig. 2 shows the total energy and magnetic moment as a function of the volume. As with the PBE GGA, the screened HSE06 gave correctly an FM bcc ground state. The calculated equilibrium lattice constant, bulk modulus, magnetic moment, and cohesive energy are summarized in Table 1 . The calculated equilibrium lattice constant $\left(a_{0}=2.906 \AA\right)$ was $1.9 \%$ larger than the experimental value $\left(a_{0}^{\text {expt }}=2.853 \AA\right)$ [36]. The calculated bulk modulus $\left(B_{0}=175 \mathrm{GPa}\right)$ was very close to the experimental value $\left(B_{0}^{\text {expt }}=168 \mathrm{GPa}\right)$ [37]. For the magnetic moment, a very large value of $2.81 \mu_{\mathrm{B}}$ was obtained, which is in contrast with the experimental value of $2.13 \mu_{\mathrm{B}}$ [38] and the PBE GGA value of $2.20 \mu_{\mathrm{B}}$ obtained in this study. The magnetization energy in the bcc phase was $2.34 \mathrm{eV}$, which is considerably larger than the PBE GGA result. A

Table 1. Equilibrium lattice constant $a_{0}$, bulk modulus $B_{0}$, magnetic moment $\mu$, cohesive energy $E_{\mathrm{b}}$, and magnetization energy $E_{\text {mag }}$ for FM bcc Fe obtained with the PBE GGA and HSE06. The other theoretical results $[6,31]$ with the GGA functional of Perdew and coworkers (PW) [33-35] are given for comparison

\begin{tabular}{lccccc}
\hline \hline XC & $\begin{array}{c}a_{0} \\
(\AA)\end{array}$ & $\begin{array}{c}B_{0} \\
(\mathrm{GPa})\end{array}$ & $\begin{array}{c}(\mu) \\
\left(\mu_{\mathrm{B}}\right)\end{array}$ & $\begin{array}{c}E_{\mathrm{b}} \\
(\mathrm{eV})\end{array}$ & $(\mathrm{eV})$ \\
\hline PBE (present work) & 2.833 & 177 & 2.20 & 4.92 & 0.48 \\
HSE06 (present work) & 2.906 & 175 & 2.81 & 3.23 & 2.34 \\
PBE $^{\mathrm{a}}$ & 2.831 & 173 & 2.18 & & \\
PW $^{\mathrm{b}}$ & 2.858 & 169 & 2.32 & & 0.52 \\
PW $^{\mathrm{c}}$ & 2.880 & 182 & 2.13 & & 0.48 \\
Experiments & $2.853^{d}$ & $168^{e}$ & $2.13^{f}$ & & \\
\hline
\end{tabular}

Reference $11,{ }^{\mathrm{b}}$ Reference 6 , ${ }^{\mathrm{c}}$ Reference $31,{ }^{\mathrm{d}}$ Reference $36,{ }^{\mathrm{e}}$ Reference 37, Reference 38

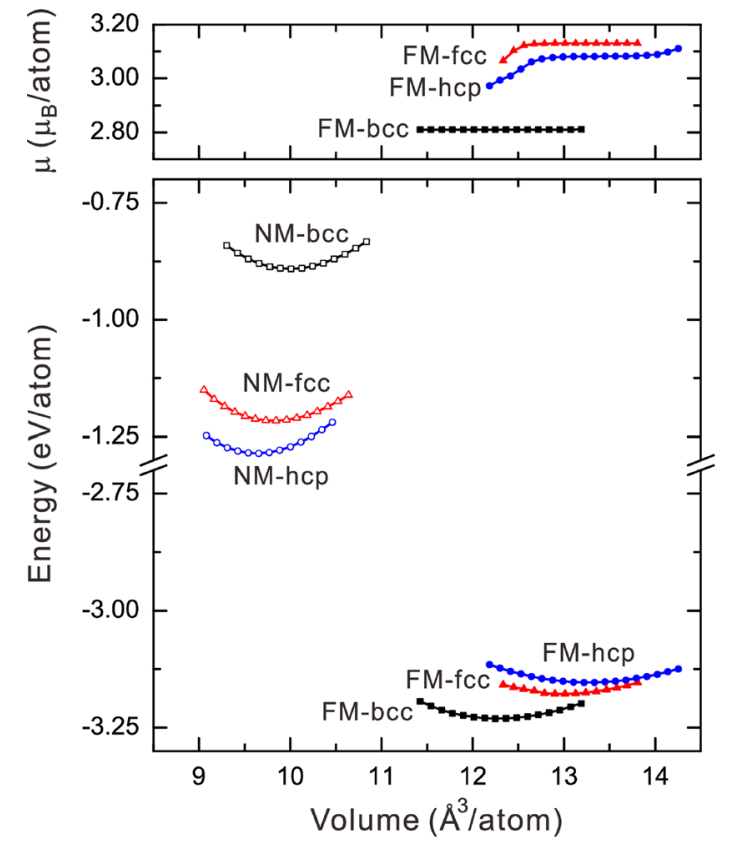

Fig. 2. (Color online) Magnetic moments and total energies as a function of volume for bcc, fcc, and hcp Fe in the NM and FM phases from screened hybrid HSE06 calculations.

cohesive energy of $3.23 \mathrm{eV}$ in the FM bcc phase was obtained, which results in a discrepancy of $1.69 \mathrm{eV}$ with respect to the PBE GGA value.

An explanation for the unusually large magnetic moment obtained by the screened hybrid HSE06 can be found in

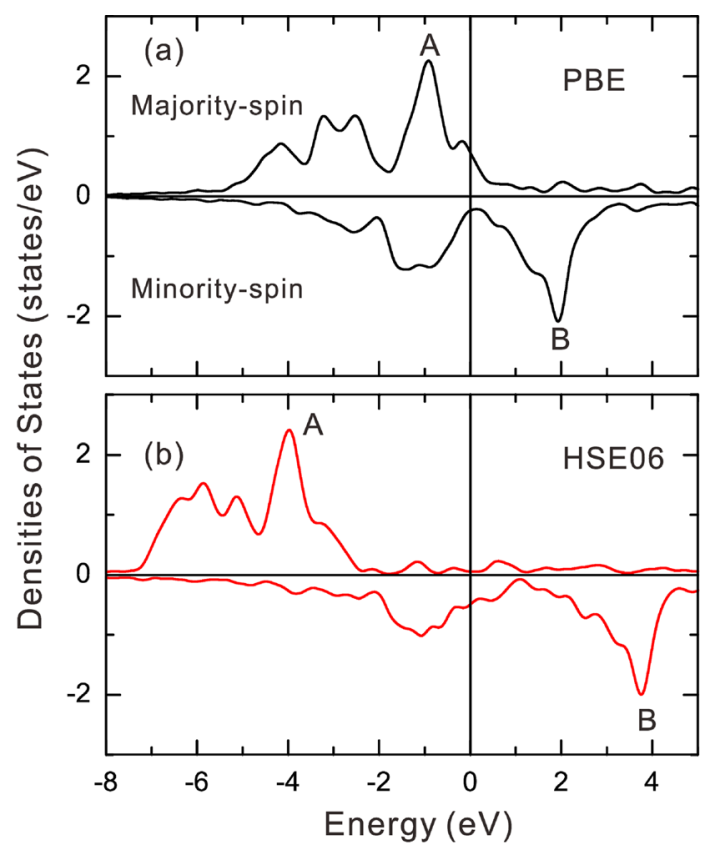

Fig. 3. (Color online) Total densities of states for FM bcc Fe obtained from the (a) PBE and (b) HSE06 calculations. The energy zero was set at the Fermi energy. 
the electronic structure of FM bcc Fe. Figs. 3(a) and 3(b) present the majority-spin (MJ-spin) and minority-spin (MN-spin) electronic densities of states (DOSs) obtained by the PBE GGA and HSE06 at each equilibrium lattice constant, respectively. To enable a direct comparison of the HSE06 result with the PBE GGA result, we used identical computational parameters of a $14 \times 14 \times 14$ kpoint mesh and a Gaussian smearing width of $0.2 \mathrm{eV}$ for the BZ integration. The DOS calculations showed significant changes in both MJ-spin and $\mathrm{MN}$-spin $\mathrm{Fe}$ states of the HSE06 in comparison with those of the PBE functional: in the case of the MJ-spin states, the peak state A was seen to move down from $-1 \mathrm{eV}$ to $-4 \mathrm{eV}$, while in the case of the MN-spin states, the peak state $\mathrm{B}$ was observed to move up from $2 \mathrm{eV}$ to $4 \mathrm{eV}$. As such, the exchange splitting increases significantly. Occupation in the MJ-spin channel increased, while the MN-spin occupation number decreased. Such a change in the electronic structure obtained by the HSE06 calculations provides a good explanation for a larger magnetic moment $\left(2.81 \mu_{\mathrm{B}}\right)$ compared with those of the PBE $\left(2.20 \mu_{\mathrm{B}}\right)$ and of the experiment $\left(2.13 \mu_{\mathrm{B}}\right)$. While, in metallic systems, the nonlocal Fock exchange term should be strongly screened, a certain amount of the nonlocal exchange term is used in hybrid functional calculations, leading to an overestimation of the exchange splitting [26]. This indicates that, to avoid an overestimation of the exchange splitting, a different treatment of the exact exchange would be required in oxide/metal systems.

\section{Summary}

We have performed total energy calculations for the ground state properties of $\mathrm{Fe}$ using the PBE GGA and screened hybrid HSE06 functionals as the exchangecorrelation functional. Both calculations provided correct results for the FM bcc ground state. The calculated equilibrium lattice constants and bulk moduli were in good agreement with experiments. However, the cohesive energy and magnetic moment significantly differed from each other. Inspection of the electronic structures of FM bcc Fe showed that the unusually small cohesive energy and large magnetic moment observed in the screened hybrid HSE06 calculations are due to an overestimation of the exchange splitting. Our results suggest that a different treatment of the exact exchange would be more desirable for oxide/metal systems.

\section{Acknowledgment}

This work was supported by the University of Seoul
2009 Research Fund (B.D.Y.).

\section{References}

[1] P. Hohenberg and W. Kohn, Phys. Rev. 136, B864 (1964).

[2] W. Kohn and L. J. Sham, Phys. Rev. 140, A1133 (1965).

[3] J. P. Perdew and K. Schmidt, in Density Functional Theory and its Application to Materials, edited by V. Van Doren, C. Van Alsenoy, and P. Geerlings, AIP, Melville, New York (2001).

[4] J. P. Perdew, J. A. Chevary, S. H. Vosko, K. A. Jackson, M. R. Pederson, D. J. Singh, and C. Fiolhais, Phys. Rev. B 46, 6671 (1992).

[5] J. P. Perdew, K. Burke, and M. Ernzerhof, Phys. Rev. Lett. 77, 3865 (1996).

[6] J.-H. Cho and M. Scheffler, Phys. Rev. B 53, 10685 (1996).

[7] B. D. Yu and M. Scheffler, Phys. Rev. Lett. 77, 1095 (1996).

[8] B. D. Yu and M. Scheffler, Phys. Rev. B 55, 13916 (1997).

[9] B. D. Yu and M. Scheffler, Phys. Rev. B 56, R15569 (1997).

[10] T. C. Leung, C. T. Chan, and B. N. Harmon, Phys. Rev. B 44, 2923 (1991).

[11] M. Kodera, T. Shishidou, and T. Oguchi, J. Phys. Soc. Jpn. 79, 074713 (2010).

[12] D. Lee and S. Hong, J. Magnetics 12, 68 (2007).

[13] W. S. Yun, G.-B. Cha, and S. C. Hong, J. Magnetics 13, 144 (2008).

[14] S.-W. Seo, Y. Y. Song, G. Rahman, I. G. Kim, M. Weinert, and A. J. Freeman, J. Magnetics 14, 137 (2009).

[15] F. Fuchs, J. Furthmüller, F. Bechstedt, M. Shishkin, and G. Kresse, Phys. Rev. B 76, 115109 (2007).

[16] J. Heyd, G. E. Scuseria, and M. Ernzerhof, J. Chem. Phys. 124, 219906 (2006).

[17] J. Heyd, G. E. Scuseria, and M. Ernzerhof, J. Chem. Phys. 118, 8207 (2003).

[18] C. Adamo and V. Barone, J. Chem. Phys. 110, 6158 (1999).

[19] H.-J. Freund, Angew. Chem. 109, 444 (1997).

[20] C. Stampfl, M. V. Ganduglia-Pirovano, K. Reuter, and M. Scheffler, Surf. Sci. 500, 368 (2002).

[21] J. Park, I. Park, and B. D. Yu, J. Korean Phys. Soc. 54, 109 (2009).

[22] J. Park and B. D. Yu, J. Phys. Soc. Jpn. 79, 074718 (2010).

[23] Y.-R. Jang, J. Park, and B. D. Yu, J. Phys. Soc. Jpn. 79, 124703 (2010).

[24] S. Yuasa, T. Nagahama, A. Fukushima, Y. Suzuki, and K. Ando, Nature Mater. 3, 868 (2004).

[25] J. Park and B. D. Yu, Phys. Rev. B 83, 144431 (2011).

[26] J. Paier, M. Marsman, K. Hummer, G. Kresse, I. C. Gerber, and J. G. Ángyán, J. Chem. Phys. 124, 154709 (2006).

[27] G. Kresse and J. Hafner, Phys. Rev. B 47, R558 (1993). 
[28] G. Kresse and J. Furthmuller, Phys. Rev. B 54, 11169 (1996).

[29] P. E. Blöchl, Phys. Rev. B 50, 17953 (1994).

[30] F. Birch, Phys. Rev. 71, 809 (1947).

[31] D. J. Singh, W. E. Pickett, and H. Krakauer, Phys. Rev. B 43, 11628 (1991).

[32] H. L. Zhang, S. Lu, M. P. J. Punkkinen, Q.-M. Hu, B. Johansson, and L. Vitos, Phys. Rev. B 82, 132409 (2010).

[33] J. P. Perdew, Phys. Rev. B 33, 8822 (1986).

[34] J. P. Perdew, Electronic Structure of Solids '91, edited by
P. Ziesche and H. Eschrig, Akademie-Verlag, Berlin (1991).

[35] J. P. Perdew, J. A. Chevary, S. H. Vosko, K. A. Jackson, M. R. Pederson, D. J. Singh, and C. Fiolhais, Phys. Rev. B 46, 6671 (1992).

[36] American Institute of Physics Handbook, edited by D. E. Gray, 3re ed., McGraw-Hill, New York (1972).

[37] C. Kittel, Introduction to Solid State Physics, 8th ed., Wiley, New York (2004).

[38] G. Y. Guo, Phys. Rev. B 55, 11619 (1997). 